OPEN ACCESS

Edited by: Giovanna Suzzi, University of Teramo, Italy

Reviewed by: Giuseppe Blaiotta, Università degli Studi di Napoli

Federico II, Italy

Maria João Fraqueza, Universidade de Lisboa, Portugal

${ }^{*}$ Correspondence: Sabine Leroy sabine.leroy@inra.fr

Régine Talon regine.talon@inra.fr

tThese authors have contributed equally to this work.

Specialty section: This article was submitted to Food Microbiology, a section of the journal Frontiers in Microbiology

Received: 31 January 2017 Accepted: 23 March 2017

Published: 06 April 2017

Citation:

Ras G, Zuliani V, Derkx P, Seibert TM, Leroy $S$ and Talon $P$ (2017) Evidence for Nitric Oxide Synthase Activity in Staphylococcus xylosus Mediating Nitrosoheme

Formation. Front. Microbiol. 8:598. doi: 10.3389/fmicb.2017.00598

\section{Evidence for Nitric Oxide Synthase Activity in Staphylococcus xylosus Mediating Nitrosoheme Formation}

\author{
Geoffrey Ras ${ }^{1,2}$, Véronique Zuliani ${ }^{2}$, Patrick Derkx ${ }^{3}$, Tim M. Seibert ${ }^{4}$, Sabine Leroy ${ }^{1 \text { *t }}$ and \\ Régine Talon ${ }^{1 * t}$
}

${ }^{1}$ Université Clermont Auvergne - INRA, MEDIS, Clermont-Ferrand, France, ${ }^{2}$ CHR. HANSEN SAS, Saint Germain les Arpajon, France, ${ }^{3}$ CHR. HANSEN A/S, Hørsholm, Denmark, ${ }^{4}$ CHR. HANSEN GmbH, Pohlheim, Germany

Staphylococcus xylosus is used as a starter culture in fermented meat products and contributes to color formation by the reduction of nitrate to nitrite. Nitrite is a food additive that is chemically turned to nitric oxide (NO) in meat but its safety has been questioned. The objective of this study was to determine the ability of NO synthase (NOS) of S. xylosus C2a to produce NO. For this purpose, a nos deletion mutant ( $\Delta$ nos) in S. xylosus was constructed and $\mathrm{NO}$ production was evaluated in a test based on its ability to form nitrosomyoglobin and nitrosoheme. Production of NO was abrogated in the $\Delta$ nos mutant under aerobic conditions and reduced about $35-40 \%$ comparing to the wild type C2a under limited oxygenation. This mutant was sensitive to oxidative stress. The expression of genes encoding catalase was modulated in the mutant with an up-regulation of katA and a down-regulation of katB and katC. The $\Delta$ nos mutant displayed high colony pigmentation after prolonged growth on agar medium. Finally, the $\Delta$ nos mutant showed no growth in minimal medium. Growth was not restored in the minimal medium by complementation with nos, but was restored by either addition of phenylalanine or complementation with $p d t$, a gene that encodes a prephenate dehydratase involved in phenylalanine biosynthesis and co-transcribed with nos. Our findings clearly demonstrate NOS-mediated NO production in S. xylosus, a meat-associated coagulase-negative Staphylococcus.

Keywords: nitric oxide synthase, nitric oxide, nitrosoheme, Staphylococcus xylosus, coagulase-negative Staphylococcus, oxidative stress

\section{INTRODUCTION}

Staphylococcus xylosus, a coagulase-negative Staphylococcus (CNS), is commonly used as starter culture to boost color development of cured meat products such as dried fermented sausages (Talon and Leroy, 2006). The typical reddish color relies on the presence of the curing salts, nitrate and nitrite. Nitrate salts are reduced to nitrite by nitrate reductase activity of $S$. xylosus (Talon et al., 1999; Gøtterup et al., 2008). Nitrite is subsequently converted by chemical reactions to nitric oxide (NO), which is able to bind to the ferrous heme-iron to form the stable red nitrosomyoglobin pigment (Gøtterup et al., 2007, 2008).

The use of the curing agents nitrate and nitrite is regulated by law, with specific indications in the United States (Code of Federal Regulations, 2016) and Europe (Commission Regulation [EU], 2011). Nowadays, the safety regarding the use of such additives on meat products is questioned. 
Nitrites can react with secondary amine groups of muscle proteins as well as compounds present in the gastrointestinal tract to form undesired $\mathrm{N}$-nitroso compounds such as nitrosamines, which could pose a health threat (Honikel, 2008). The meat industry is therefore looking for alternatives to decrease nitrate/nitrite in the production of meat products.

Staphylococcus xylosus has been shown to convert metmyoglobin to nitrosomyoglobin in culture medium, in salami (Morita et al., 1998) and in raw meat batter (Li et al., 2013, 2016), without addition of nitrate or nitrite. NO production has been suggested to be linked to NO synthase (NOS) activity. NOS catalyzes the production of NO from l-arginine and was initially described in mammals (Alderton et al., 2001). Homologs of the oxygenase domain of mammalian NOS were identified in Gram-positive bacteria. NOS is involved in the production of NO by Bacillus subtilis (Gusarov and Nudler, 2005; Gusarov et al., 2008), Bacillus anthracis (Shatalin et al., 2008), Bacillus cereus (Montgomery et al., 2010), Geobacillus stearothermophilus (Kabir et al., 2008), Streptomyces turgidiscabies (Kers et al., 2004), Deinococcus radiodurans (Patel et al., 2009), and Staphylococcus aureus (van Sorge et al., 2013). S. aureus NOS is involved in resistance to oxidative stress and antibiotics, and virulence (Vaish and Singh, 2013; van Sorge et al., 2013; Sapp et al., 2014). In all sequenced $S$. aureus genomes, the nos gene is part of a cluster containing another gene encoding a prephenate dehydratase, which is involved in phenylalanine biosynthesis. This genetic organization appears to be unique to staphylococcal genomes (Sapp et al., 2014). The nos genes have been identified in many staphylococci (van Sorge et al., 2013; Sánchez-Mainar et al., 2014; Sapp et al., 2014), but NOSmediated NO production has only been characterized in the pathogenic S. aureus. The contribution of the NOS activity of S. xylosus to formation of nitrosomyoglobin remains to be demonstrated.

In an attempt to determine the ability of $S$. xylosus NOS to produce $\mathrm{NO}$, we generated a nos deletion mutant in the S. xylosus C2a strain. NO production in S. xylosus C2a and mutants was investigated using an assay based on the conversion of metmyoglobin to red pigment derivatives followed by an extraction of nitrosoheme. We also assessed the sensitivity of the mutants to oxidative stress.

\section{MATERIALS AND METHODS}

\section{Bacterial Strains and Growth Conditions}

The bacterial strains and plasmids used in this study are listed in Table 1. Experiments were performed using S. xylosus strain C2a or its isogenic mutants. S. xylosus was routinely cultured in Tryptic Soy Broth (TSB, Difco) under aerobic conditions (1:10 volume to flask ratio, $150 \mathrm{rpm}$ ) or on Tryptic Soy Agar (TSA, Difco) at $30^{\circ} \mathrm{C}$. When required, strains were grown in a minimal medium (MX) as already described (Fiegler and Brückner, 1997). The MX medium, when needed, was supplemented with amino acids $(100 \mathrm{mg} / \mathrm{L})$. For genetic manipulation, Escherichia coli was cultured in Luria-Bertani (LB, Difco) broth with shaking aeration $(150 \mathrm{rpm})$ or on LB agar (Difco) at $37^{\circ} \mathrm{C}$. Antibiotics, when required to maintain plasmids or select recombinants, were added at the following concentrations: for E. coli, $100 \mu \mathrm{g} / \mathrm{mL}$ ampicillin; for S. xylosus, $20 \mu \mathrm{g} / \mathrm{mL}$ chloramphenicol, or 2.5 to $10 \mu \mathrm{g} / \mathrm{mL}$ erythromycin. All chemicals were from SigmaAldrich.

To compare growth kinetics under microaerobic conditions, overnight cultures of $S$. xylosus were diluted to an optical density (OD) of 0.05 to 0.10 at $600 \mathrm{~nm}$ and incubated in 100 -well microtiter plates with shaking at $30^{\circ} \mathrm{C}$ in a Bioscreen $\mathrm{C}$ plate reader (Labsystems France) while the turbidity was monitored every $30 \mathrm{~min}$ for $24 \mathrm{~h}$. Three independent experiments were done for each set of conditions.

\section{DNA Manipulation}

Genomic DNA from S. xylosus was prepared from overnight cultures. Briefly, cells were resuspended in Tris-EDTA-sucrose buffer containing $0.1 \mathrm{mg} / \mathrm{mL}$ lysostaphin (Sigma-Aldrich) and incubated for $30 \mathrm{~min}$ at $37^{\circ} \mathrm{C}$. Cells were lysed with sodium dodecyl sulfate and treated with RNase A. Following extraction with phenol-chloroform-isoamyl alcohol (25/24/1) and chloroform, DNA was precipitated with ethanol and resuspended in Tris-EDTA buffer ( $\mathrm{pH} \mathrm{8.0)}$. Plasmid DNA from E. coli was isolated using the NucleoSpin Plasmid QuickPure kit (Macherey-Nagel). Restriction digests were performed using high-fidelity restriction enzymes (New England Biolabs). Ligations were performed using T4 DNA ligase (Roche). Amplifications were performed using Phusion High-Fidelity DNA Polymerase (New England Biolabs) for cloning and GO Taq DNA polymerase (Promega) for standard purposes. PCR products were visualized by $0.8 \%$ agarose gel electrophoresis and ethidium bromide staining, and imaged on a Gel Doc 2000 (Bio-Rad). As required, they were purified using the QIAquick Gel Extraction kit (Qiagen). DNA sequencing was performed by GATC Biotech (Mulhouse, France).

\section{Construction of S. xylosus NO Synthase Mutant}

The oligonucleotides used in this study were designed based on the genome sequence of $S$. xylosus C2a (GenBank accession no. LN554884) and are listed in Table 2. A chromosomal nos disruption mutant was constructed by deletion of the nos coding sequence corresponding to amino acids 11 to 345 (out of 354) and insertion of an erythromycin resistance cassette $(\mathrm{ermB})$ using the temperature-sensitive vector pBT2. Briefly, a 756-bp upstream fragment and a 783-bp downstream fragment were separately amplified by using the genomic DNA of $S$. xylosus $\mathrm{C} 2 \mathrm{a}$ as template. The ermB gene was amplified from the pEC4 vector generating an amplicon of 1,366-bp. The three purified PCR products were annealed by overlapping PCR using the outside primers and inserted into pBT2 using appropriate restriction enzymes. The resulting plasmid was constructed in competent E. coli TOP10 and introduced into S. xylosus C2a by electroporation as described (Götz et al., 1983; Brückner, 1997). Gene replacement was allowed to take place by incubating the temperaturesensitive plasmid at $42^{\circ} \mathrm{C}$ as described (Brückner, 1997). 
TABLE 1 | List of strains and vectors used in this study.

\begin{tabular}{|c|c|c|}
\hline Strain or plasmid & Relevant characteristics & Source or reference \\
\hline \multicolumn{3}{|c|}{ Staphylococcus xylosus } \\
\hline $\mathrm{C} 2 \mathrm{a}$ & Derived from the type strain DSM20267 and genetically transformable strain & Götz et al., 1983, LN554884 \\
\hline$\Delta$ nos & Isogenic mutant of C2a deleted of the SXYL_00923 gene, $\Delta$ nos:::ermB & This study \\
\hline$\Delta$ nospRBnos & $\Delta$ nos complemented with nos & This study \\
\hline$\Delta$ nospRBpdt & Anos complemented with pdt (SXYL_00922) & This study \\
\hline$\Delta$ nospRBnospdt & $\Delta$ nos complemented with nospdt & This study \\
\hline C2apRB473 & C2a containing empty vector pRB473 & This study \\
\hline C2apRB474 & C2a containing empty vector pRB474 & This study \\
\hline$\Delta n o s p R B 473$ & $\Delta$ nos containing empty vector pRB473 & This study \\
\hline$\Delta$ nospRB474 & $\Delta$ nos containing empty vector pRB474 & This study \\
\hline \multicolumn{3}{|l|}{ Escherichia coli } \\
\hline TOP10 & Competent strain for plasmid transformation & Invitrogen \\
\hline \multicolumn{3}{|l|}{ Plasmids } \\
\hline pBT2 & E. coli-S. xylosus thermosensitive shuttle vector, $\mathrm{Ap}^{R} \mathrm{Cm}^{R}$ & Brückner, 1997 \\
\hline pEC4 & pBluescript KS + derivative. Source of ermB gene (EmR). ApR & Brückner, 1997 \\
\hline pRB473 & Shuttle vector $A p^{R}$ and $\mathrm{Cm}^{\mathrm{R}}$ & Brückner, 1992 \\
\hline pRB474 & Shuttle vector $A p^{R}$ and $\mathrm{Cm}^{\mathrm{R}}$ & Brückner, 1992 \\
\hline pBT $\Delta$ nos & pBT2, SXYL_00923 [13-317]::ermB shuttle vector & This study \\
\hline pRBnos & pRB473 derivate for expression of nos & This study \\
\hline pRBpdt & pRB474 derivate for expression of pdt & This study \\
\hline pRBnospdt & pRB473 derivate for expression of nos and pdt & This study \\
\hline
\end{tabular}

Successful C2a $\Delta$ nos mutant was screened for resistance to erythromycin and sensitivity to chloramphenicol, indicating loss of the pBT2 backbone, and this was confirmed by PCR and sequencing.

\section{Creation of Complementation Plasmids}

Complementation of the nos mutation was performed by amplifying the nos gene with its putative promoter. The corresponding amplicon was cloned into the shuttle vector pRB473 using appropriate enzymes, generating pRBnos. Similarly, an nos-pdt complementation vector ( $\mathrm{pRBnospdt}$ ) was constructed. A complementation vector pRBpdt was constructed, placing the expression of $p d t$ under the constitutive promoter of pRB474. All PCR-amplified fragments were verified by sequencing. The resulting plasmids were constructed in competent E. coli TOP10 and introduced into S. xylosus $\mathrm{C} 2 \mathrm{a} \Delta$ nos by electroporation according to an established protocol (Brückner, 1997).

\section{Metmyoglobin Conversion and Assessment of Nitrosoheme Formation}

A $20 \mathrm{mg} / \mathrm{mL}$ metmyoglobin solution was freshly prepared from equine heart myoglobin (Sigma-Aldrich) as described (Gündoğdu et al., 2006). Overnight S. xylosus cultures were inoculated in TSB supplemented with $2 \mathrm{mg} / \mathrm{mL}$ metmyoglobin to a final $\mathrm{OD}_{600 \mathrm{~nm}}$ of $0.5(8 \log \mathrm{CFU} / \mathrm{mL})$. Cultures were incubated either under limited oxygenation (covered with mineral oil and without stirring) or under aerobic conditions as described above. After $24 \mathrm{~h}$ of incubation at $30^{\circ} \mathrm{C}, \mathrm{OD}_{600 \mathrm{~nm}}$ and $\mathrm{pH}$ of the cultures were measured and cells were serial-diluted and plated on TSA for enumeration. Cultures were centrifuged and supernatants were used to measure the absorbance spectrum between 500 and $600 \mathrm{~nm}$ (BioMate 3, Thermo Fisher Scientific) using as control a solution of sterile TSB supplemented with metmyoglobin and incubated in the same conditions. Finally, supernatants were treated with acetone 1:4(v/v) to extract the nitrosoheme from nitrosomyoglobin, and the absorbance spectrum was acquired between 450 and $640 \mathrm{~nm}$ (V-770 UV/visible, Jasco). Sterile TSB supplemented with metmyoglobin and extracted with acetone was used as control. Experiments were carried out in three independent biological replicates.

\section{Analysis of colony pigmentation}

After overnight growth in TSB, S. xylosus strains were plated on TSA. After 2 days of growth (when pigmentation is strongest), $100 \mathrm{mg}$ of each strain was collected from plates and pigments were extracted and quantified according the method of Morikawa et al. (2001). Briefly, cells were washed once in water, centrifuged for $5 \mathrm{~min}$ at $15,000 \mathrm{~g}$, re-suspended in $1 \mathrm{~mL}$ of methanol and heated for $5 \mathrm{~min}$ at $55^{\circ} \mathrm{C}$. Cells were centrifuged and the supernatant containing pigment was retrieved. The absorbance spectrum of methanol-extracted pigment was measured between 400 and $600 \mathrm{~nm}$ to evaluate carotenoid content. A peak at $\lambda=460 \mathrm{~nm}$ was detected and results are expressed as relative optical density at $460 \mathrm{~nm}$ with normalization to C2a. Experiments were performed with three independent biological replicates.

\section{Survival Experiments after Exposure to Different Stresses}

The S. xylosus strains were grown in TSB under aerobic conditions. To assess sensitivity to oxidative stress, overnight 
TABLE 2 | Oligonucleotides used in this study.

\begin{tabular}{|c|c|c|}
\hline Name & Primer Sequence $\left(5^{\prime} \text { to } 3^{\prime}\right)^{\mathrm{a}, \mathrm{b}}$ & Used for \\
\hline Up923F1-EcoRl & CGGAATTCGACGCGTACCAAATTCCATT & Amplification of sequence upstream nos \\
\hline Up923R1-ErmF & CACAATAGAGAGATGTCACCTGTCGATAAAGGATTCGCTTC & \\
\hline Dn923F2-ErmR & GGTATACTACTGACAGCTTCCAACAGAAGCTACTGGTTGTCCA & Amplification of sequence downstream nos \\
\hline Dn923R2-BamHI & CGGGATCCGGTGTGTCTGCTTGGACAAA & \\
\hline ErmF-Up923R1 & GAAGCGAAATCCTTTATCGACAGGTGACATCTCTCTATTGTG & Amplification of ermB \\
\hline ErmR-Dn923F2 & TGGACAACCAGTAGCTTCTGTTGGAAGCTGTCAGTAGTATACC & \\
\hline Up923F3 Dn923R3 & TCCTGCTCGCACATTACTTG CGTCAGGTATCTTGTTGCTCA & Control of nos::erm construction \\
\hline Up923PF5-Sall & ACGCGTCGACGTGCTTGATAGCACATGAAAAAGGA & Complementation of nos in pRB473 \\
\hline Dn923R5-EcoRl & CGGAATTCGGGTCGTTAATGAAATGGACA & \\
\hline Up923PF5-Sall & ACGCGTCGACGTGCTTGATAGCACATGAAAAAGGA & Complementation of nos-pdt in pRB473 \\
\hline Dn922-EcoRl & CGGAATTCTTGCTCATTAGGTTGTGCTAATTC & \\
\hline Up922-Sall & ACGCGTCGACGTAAGGGGTAACGTCAAATGAAA & Complementation of pdt in pRB474 \\
\hline Dn922-EcoRl & CGGAATTCTTGCTCATTAGGTTGTGCTAATTC & \\
\hline 923F4 922R1 & AAGCGAAATCCTIATCGACAC CTAATGGCAGGCCCAATAGA & Co-transcription nos-pdt \\
\hline 923F2 923R2 & TGCAGAAGCGTTTGAATTTG GCTTCGATGCAGTGAGATGA & qRT-PCR of nos (SXYL_00923) \\
\hline 2505F 2505R & $\begin{array}{l}\text { CGTCATCTTCACGAAAGTCATATTC } \\
\text { CGCTAGTACACATTATTATCCAATAG }\end{array}$ & qRT-PCR of katA (SXYL_2505) \\
\hline $1551 F 1551 R$ & ATTCGTGGATTCGCATAAAAG AGCTTCTGGTAGTGACGT & qRT-PCR of katB (SXYL_1551) \\
\hline 2533F 2533R & TTCGATCATGAACGTATACCA GTGTCTGGTGAACC ПTAGAG & qRT-PCR of katC (SXYL_2533) \\
\hline 1303F 1303R & CGCAGCAGTAGAAGGAACTG ATGTCCACCGCCATTATTGC & $\begin{array}{l}\text { qRT-PCR of the housekeeping gene sod } \\
\text { (SXYL_1303) and control of DNA contamination } \\
\text { in RNA extracts }\end{array}$ \\
\hline
\end{tabular}

a Underlined regions represent restriction enzyme sequences.

bPrimer containing overlapping end (in bold).

cultures were diluted to an $\mathrm{OD}_{600} \mathrm{~nm}$ of 0.05 with fresh medium and incubated until $\mathrm{OD}_{600} \mathrm{~nm}$ was approximately 1 . One milliliter of each culture was collected for serial dilutions and determination of colony-forming units (CFU)/mL on TSA plates. The remaining culture was treated with $150 \mathrm{mM}$ hydrogen peroxide (Sigma-Aldrich) and incubated for $1 \mathrm{~h}$ at $30^{\circ} \mathrm{C}$. $4,000 \mathrm{U} / \mathrm{mL}$ of catalase (Sigma-Aldrich) was added to quench residual $\mathrm{H}_{2} \mathrm{O}_{2}$ as described (Barrière et al., 2002). Cells were serial-diluted and plated in duplicate on TSA for enumeration of CFU from surviving cells.

To evaluate the impact of saline and acid stresses, overnight cultures of $S$. xylosus strains were freshly diluted (1/100) in TSB and grown under aerobic conditions at $30^{\circ} \mathrm{C}$ until $\mathrm{OD}_{600} \mathrm{~nm}$ was approximately 0.3 . Strains were diluted in $\mathrm{NaCl}$-supplemented TSB (10 and $20 \% \mathrm{NaCl}$ ) or diluted in TSB with adjusted $\mathrm{pH}(5.0$ and 6.0$)$ to an $\mathrm{OD}_{600} \mathrm{~nm}$ of 0.1 and incubated in 100-well microtiter plates with shaking at $30^{\circ} \mathrm{C}$ in a Bioscreen $\mathrm{C}$ plate reader, where turbidity was monitored every $30 \mathrm{~min}$ for 20 hours. Assays were performed with two independent cultures for each strain.

\section{Total RNA Extraction and Reverse Transcription}

Staphylococcus xylosus strains were grown in TSB under aerobic conditions for $6 \mathrm{~h}$ and $24 \mathrm{~h}$. Cell pellets were immediately frozen in liquid nitrogen to stabilize the bacterial RNA and stored at $-80^{\circ} \mathrm{C}$. For RNA extraction, cell pellets were thawed on ice and resuspended in $500 \mu \mathrm{L}$ of cold Tris-EDTA buffer. Samples were transferred into tubes containing $600 \mathrm{mg}$ zirconia-silica beads (0.1 mm diameter), $50 \mu \mathrm{L}$ of sodium dodecyl sulfate (10\%), $500 \mu \mathrm{L}$ of acid phenol and $3.5 \mu \mathrm{L}$ of $\beta$-mercaptoethanol. Cells were disrupted in a FastPrep ${ }^{\mathrm{TM}}$ machine (MP Biomedicals). After addition of $200 \mu \mathrm{L}$ of chloroform and centrifugation, the aqueous phase containing RNA was purified with the Nucleospin 
RNAII kit (Macherey Nagel) according to the manufacturer's instructions. A supplementary treatment was performed with Turbo DNAse (Ambion) to remove residual DNA. Absence of contaminating DNA was checked by PCR targeting the sod gene (Table 2). Total RNA isolated was quantified using a Nanodrop 1000 (Thermo Fisher Scientific). RNA samples were stored at $-80^{\circ} \mathrm{C}$. RNA isolated from three independent biological replicates for each strain or condition (wild-type and its mutants at 6 and $24 \mathrm{~h}$ ) was reverse-transcribed to cDNA with a SuperScript Reverse Transcriptase kit following the manufacturer's instructions (Invitrogen).

\section{Evaluation of nos-pdt Co-transcription}

To determine if the two adjacent genes, nos and $p d t$, were co-transcribed, cDNA from the mRNA species transcribed from C2a 24-h cultures was subject to PCR using a pair of primers targeting the nos-pdt junction region (Table 2). The conditions for the amplification were $5 \mathrm{~min}$ at $95^{\circ} \mathrm{C}$, followed by 25 cycles of $30 \mathrm{~s}$ at $95^{\circ} \mathrm{C}, 30 \mathrm{~s}$ at $60^{\circ} \mathrm{C}$ and $120 \mathrm{~s}$ at $72^{\circ} \mathrm{C}$ and finally $5 \mathrm{~min}$ at $72^{\circ} \mathrm{C}$. As controls, this amplification was also performed on $\mathrm{C} 2 \mathrm{a}$ genomic template DNA and no template sample.

\section{Quantitative Real-time PCR}

Expression of genes of interest was carried out by quantitative real-time PCR (qRT-PCR) using iQ ${ }^{\mathrm{TM}} \mathrm{SYBR}^{\circledR}$ Green Supermix (Bio-Rad) and the MasterCycler RealPlex (Eppendorf). Thermal cycling consisted of $30 \mathrm{~s}$ at $95^{\circ} \mathrm{C}$, followed by 40 cycles of $15 \mathrm{~s}$ at $95^{\circ} \mathrm{C}$ and $60 \mathrm{~s}$ at $60^{\circ} \mathrm{C}$. The primers used are listed in Table 2. The relative fold change of gene expression, using measured sod housekeeping gene expression, was determined by the Livak $\left(2^{-\Delta \Delta \mathrm{Ct}}\right)$ method (Livak and Schmittgen, 2001).

\section{Statistics}

The data were analyzed by using GraphPad Prism software (version 5.01). The significance of experimental differences in bacterial growth, formation of myoglobin derivatives, and colony pigment production were analyzed by one-way analysis of variance (ANOVA) with Tukey's multiple comparison.

\section{RESULTS}

\section{NOS Contributes to Growth under Aerobic Conditions}

To define the role of NOS activity in S. xylosus C2a, a mutant strain $(\Delta n o s)$ was created by insertion of an erythromycin resistance gene cassette into the nos coding sequence. Deletion of the nos gene was confirmed by PCR analysis and sequencing (data not shown). When grown in TSB under aerobic conditions, the $\Delta$ nos mutant displayed a slight growth defect compared with the wild type (Figure 1A). Complementation of the $\Delta$ nos mutant with a plasmid expressing the nos gene $(\Delta$ nospRBnos $)$ showed restored growth (Figure 1B). The wild type and the $\Delta$ nos and nos complemented mutants did not differ in growth in microtiter plates under microaerobic conditions (Supplementary Figure S1).

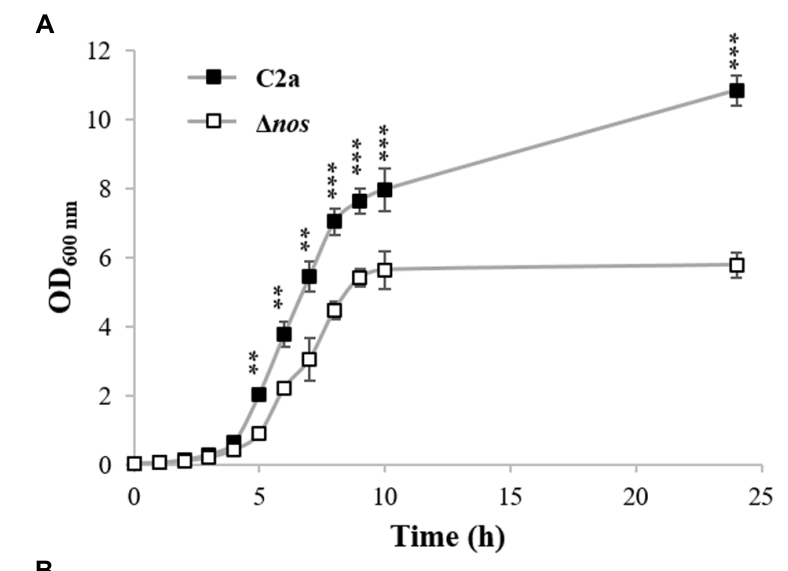

B

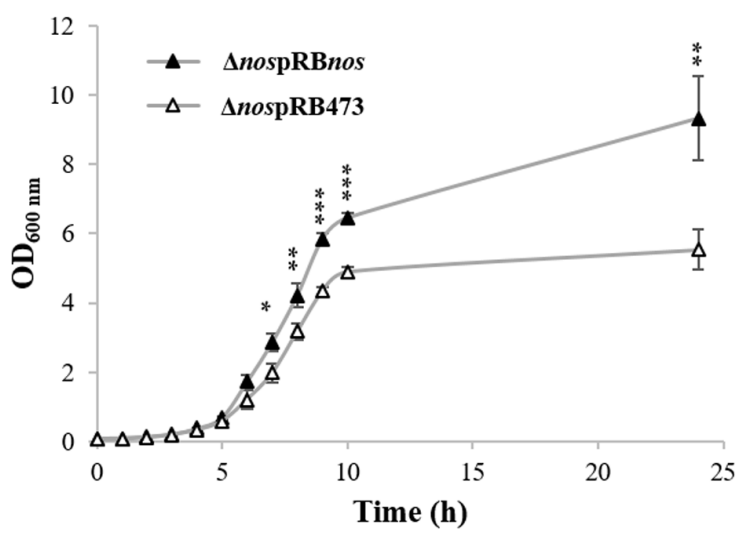

FIGURE 1 | Growth of Staphylococcus xylosus strains in TSB under aerobic conditions. (A) S. xylosus C2a and $\Delta$ nos. (B) $\Delta$ nospRBnos and $\Delta$ nospRB473 with chloramphenicol. Data represent means $\pm \mathrm{SD}$ from $n=3$ independent biological replicates. ${ }^{*} p<0.05$; ${ }^{* *} p<0.01$; ${ }^{* * *} p<0.001$.

\section{PDT But Not NOS Is Required for Growth in Minimal Medium}

Growth was measured in minimal medium under microaerobic conditions for the wild-type C2a, the $\Delta$ nos mutant and the nos complemented $\Delta$ nos mutant (Figure 2A). The $\Delta$ nos mutant was not able to grow in this minimal medium and growth was not restored by plasmid complementation with the nos gene (Figure 2A). Since the nos gene is in a cluster with the $p d t$ gene, we tested the hypothesis that both genes were cotranscribed (Supplementary Figure S2). RT-PCR experiments were carried out using a primer annealing specifically with nos and a primer annealing specifically with $p d t$ (Table 2). Amplification of a 1,421-bp fragment indicated a co-transcription of both nos and pdt (data not shown). We therefore speculated that the deletion of nos and the insertion of ermB had a polar effect on the downstream gene $p d t$, leading to a frameshift in the $p d t$ open reading frame (ORF). The $p d t$ gene encodes a prephenate dehydratase (EC4.2.1.51), which catalyzes the penultimate reaction in the phenylalanine biosynthesis. Supplementation with phenylalanine restored the growth of the $\Delta$ nos and the nos complemented ( $\Delta$ nospRBnos) mutants 

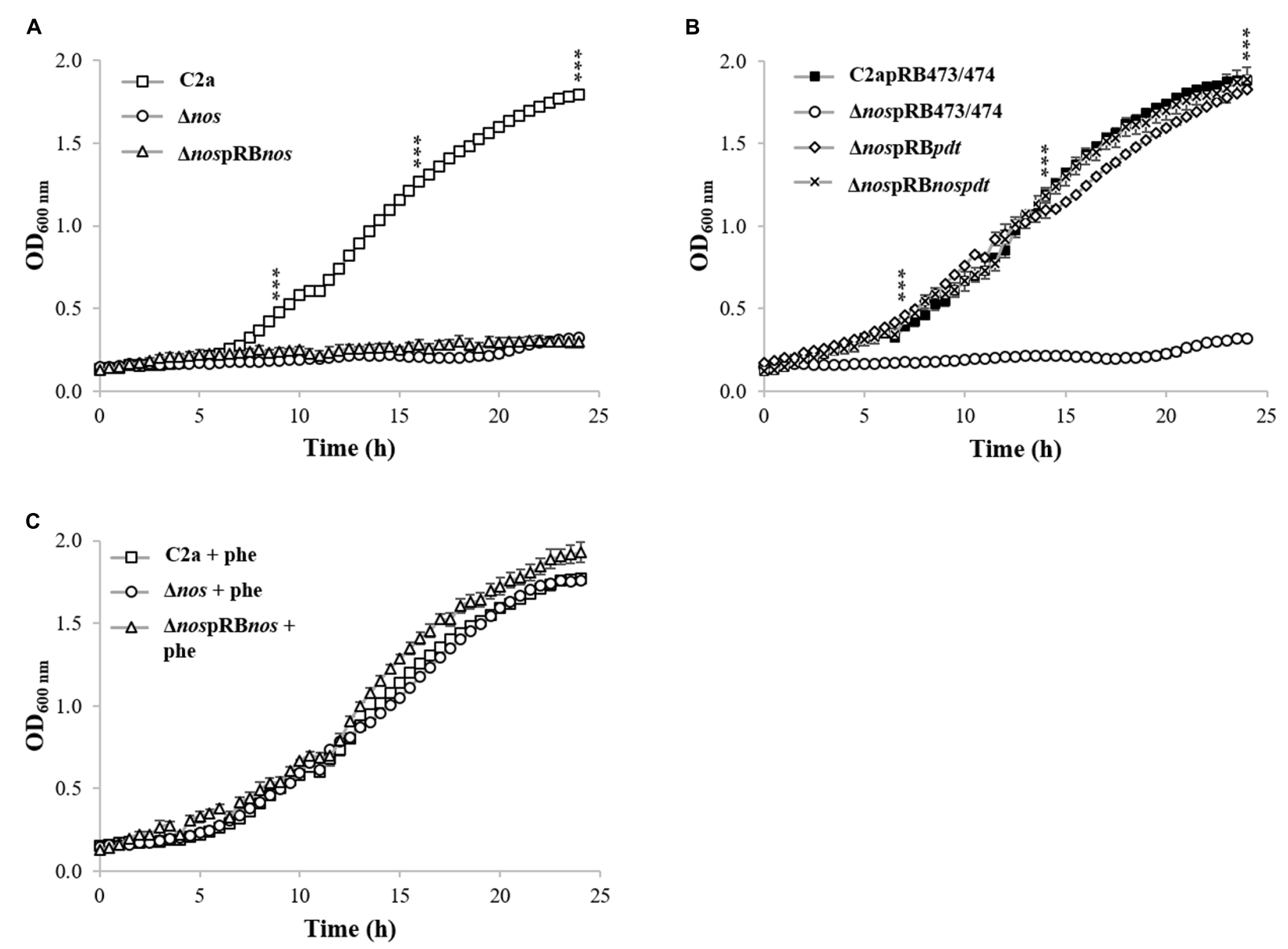

FIGURE 2 | Growth of S. xylosus strains in chemically defined minimal medium in the Bioscreen Assay. (A) Growth of S. xylosus C2a, $\triangle$ nos, and $\Delta$ nospRBnos, (B) Growth of C2a, $\Delta$ nos and $\Delta$ nospRBnos with phenylalanine (phe), (C) Growth of C2apRB473 (identical growth as C2apRB474), $\Delta$ nospRB473 (identical growth as $\Delta$ nospRB474), $\Delta$ nospRBpdt and $\Delta$ nospRBnospdt. Data represent means $\pm \mathrm{SD}$ from $n=3$ independent biological replicates. ${ }^{* * *} p<0.001$.

(Figure 2B), while no restoration of growth was observed with other amino acids such as tryptophan or tyrosine, which, like phenylalanine, are derived from chorismate (data not shown). Moreover, complementation of the $\Delta$ nos mutant with $p d t$ $(\Delta n o s \mathrm{pRB} p d t)$ or with the nos-pdt operon ( $\Delta$ nospRBnospdt) restored growth to the wild-type $\mathrm{C} 2$ a level (Figure 2C).

\section{NOS Contributes to NO Production}

The formation of the red myoglobin derivatives occurred for S. xylosus C2a under limited oxygenation (Figure 3A) and aerobic conditions (Figure 3B), where different forms of myoglobin came from the conversion of metmyoglobin (brown) to some red derivatives, such as oxymyoglobin and nitrosomyoglobin. The red pigment content was sharply decreased, but not completely abolished, in the $\Delta$ nos mutant compared with the wild type, and was restored to the wildtype level in the nos complemented $\Delta$ nos mutant under both conditions (Figure 3). Under limited oxygenation after $24 \mathrm{~h}$ of incubation, the cellular population was close to initial level, 8 $\log \mathrm{CFU} / \mathrm{mL}$, for C2a $(7.8 \pm 0.04), \Delta$ nos mutant $(7.6 \pm 0.40)$ and nos complemented $\Delta$ nos mutant (7.2 \pm 0.01$)$. Under aerobic conditions, the red pigment level was higher (Figure 3B) and the cellular population reached $9 \log \mathrm{CFU} / \mathrm{mL}$ for $\mathrm{C} 2 \mathrm{a}(9.1 \pm 0.04)$, $\Delta$ nos mutant $(9.2 \pm 0.20)$ and $\Delta$ nos complemented mutant $(9.2 \pm 0.10)$.

To estimate the production of nitrosomyoglobin, the nitrosoheme of culture supernatants from S. xylosus C2a and its mutants was extracted and measured (Figure 4). Under limited oxygenation (Figure 4A), where the $\mathrm{pH}$ of cultures was about 6.0 , the nitrosoheme formation of the $\Delta$ nos mutant was reduced $\left(\mathrm{A}_{540 \mathrm{~nm}}=0.060 \pm 1.1 \mathrm{E}-03\right)$ compared with the wild type $\left(\mathrm{A}_{540 \mathrm{~nm}}=0.090 \pm 7.1 \mathrm{E}-04\right)$ and the nos complemented $\Delta$ nos mutant $\left(\mathrm{A}_{540 \mathrm{~nm}}=0.090 \pm 1.1 \mathrm{E}-03\right)$. Under aerobic conditions (Figure 4B), where the $\mathrm{pH}$ of the cultures was about 8.0, maximum absorption shifted, as described (Yu et al., 2016), to $\lambda_{580 \mathrm{~nm}}$ instead of $\lambda_{540 \mathrm{~nm}}$. In these conditions, no nitrosoheme was formed for the $\Delta$ nos mutant $\left(\mathrm{A}_{580} \mathrm{~nm}<0.005\right)$ compared with the wild type $\left(\mathrm{A}_{580} \mathrm{~nm}=0.060 \pm 2.8 \mathrm{E}-03\right)$ and the nos complemented $\Delta$ nos mutant $\left(\mathrm{A}_{580 \mathrm{~nm}}=0.080 \pm 1.4 \mathrm{E}-03\right)$.

The $\Delta$ nos mutant and the $p d t$ complemented $\Delta$ nos mutant had the same activity, while for the nospdt complemented $\Delta$ nos mutant the formation of red myoglobin derivatives was restored 


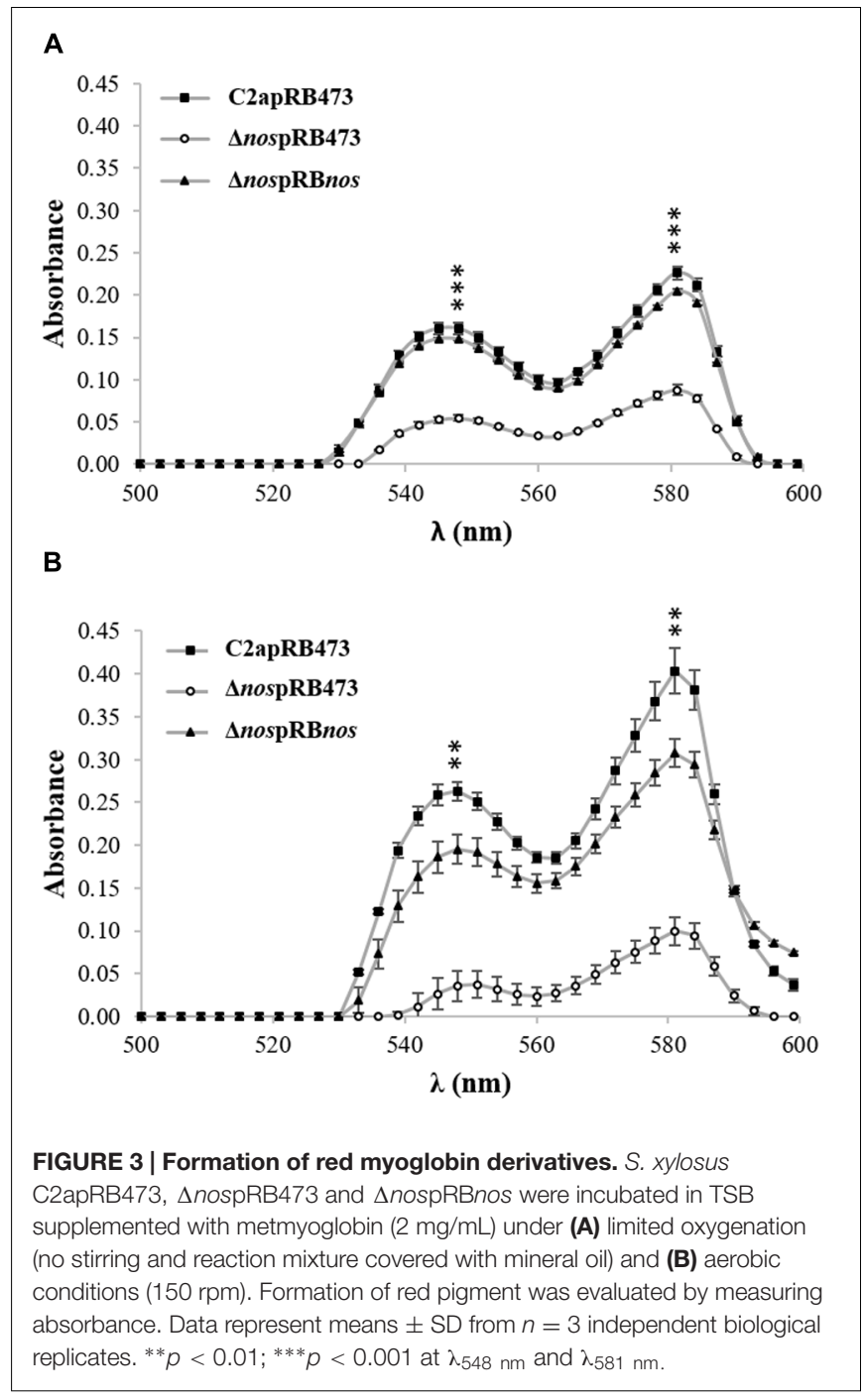

to the wild-type level (data not shown). These results clearly demonstrated the contribution of NOS and not PDT to the production of $\mathrm{NO}$ in S. xylosus $\mathrm{C} 2 \mathrm{a}$.

\section{NOS Affects Colony Pigmentation}

When grown on TSA plates, the $\Delta$ nos mutant displayed enhanced pigmentation relative to the wild type. This pigmentation returned to wild-type level upon complementation with the nos and nos-pdt genes, but not with the pdt gene alone (Figure 5A). Pigments of the wild type and the $\Delta$ nos and $\Delta$ nospRBnos mutants were extracted and absorbance at $460 \mathrm{~nm}$ was measured. Production of carotenoid pigment in the $\Delta$ nos mutant was significantly higher than in the wild type, and returned to the wild-type level in the nos complemented mutant (Figure 5B). These results clearly demonstrated that solely NOS was involved in the pigmentation phenotype. This difference in carotenoid pigment production between the wild type and the $\Delta$ nos mutant was, however, not observed with the bacterial pellets after liquid cultures in TSB, even after prolonged growth for several days (data not shown).

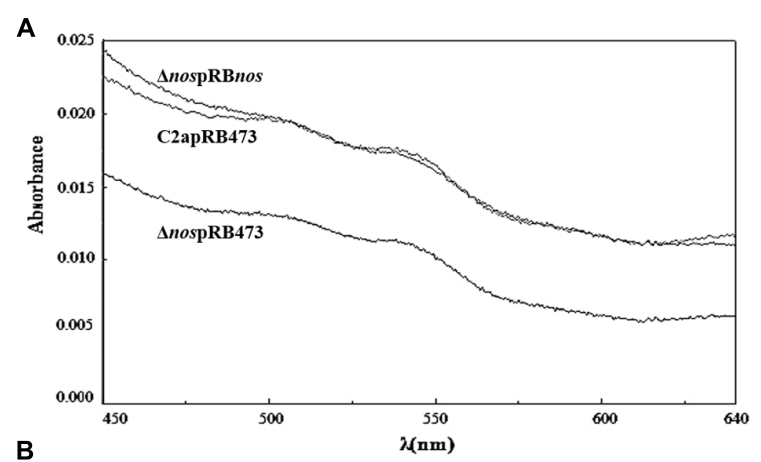

B

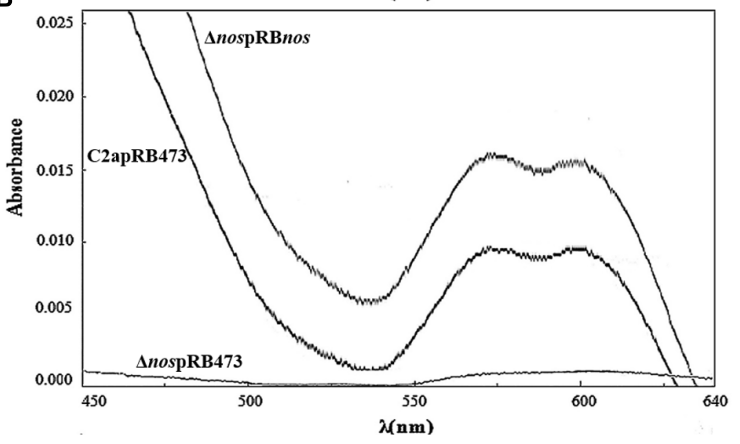

FIGURE 4 | Evidence of nitrosoheme. Nitrosoheme was extracted from nitrosomyoglobin with $80 \%$ acetone from supernatants of strains grown in TSB supplemented with metmyoglobin and inoculated under (A) limited oxygenation (no stirring and reaction mixture covered with mineral oil) and (B) aerobic conditions (150 rpm). Curves show spectra representative of three biological replicates.

\section{NOS Protects Specifically against Peroxide Stress}

Stress challenge assays with $150 \mathrm{mM} \mathrm{H} \mathrm{H}_{2} \mathrm{O}_{2}$ were performed on the wild-type $\mathrm{C} 2 \mathrm{a}$ and the $\Delta$ nos and nos complemented $\Delta$ nos mutants to investigate a possible oxidative stress-sensitive phenotype in the $\Delta$ nos mutant. After 1-h treatment, the $\Delta$ nos mutant presented a significant reduction in cell viability $(5 \mathrm{log})$ compared with the wild type (Figure 6). The phenotype was restored to the wild-type level in the nos complemented mutant (Figure 6). To evaluate the specificity of NOS in protecting against oxidative stress, stress challenge assays in saline and acid conditions were performed on the wild type and the $\Delta$ nos and nos complemented $\Delta$ nos mutants (Supplementary Figure S3). As the salt concentration increased (Supplementary Figure S3A) or the $\mathrm{pH}$ decreased (Supplementary Figure S3B), bacterial growth was affected and decreased for all strains with no significant differences between strains. These results demonstrated that NOS was not required to overcome those stresses.

\section{NOS Modulates Catalase Gene Expression}

To determine whether increased $\mathrm{H}_{2} \mathrm{O}_{2}$-sensitive phenotype and defects in growth under aerobic conditions of the $\Delta$ nos mutant were due to differential expression of catalase genes, we evaluated expression of the $k a t A, k a t B$, and $k a t C$ genes by qRT-PCR. 


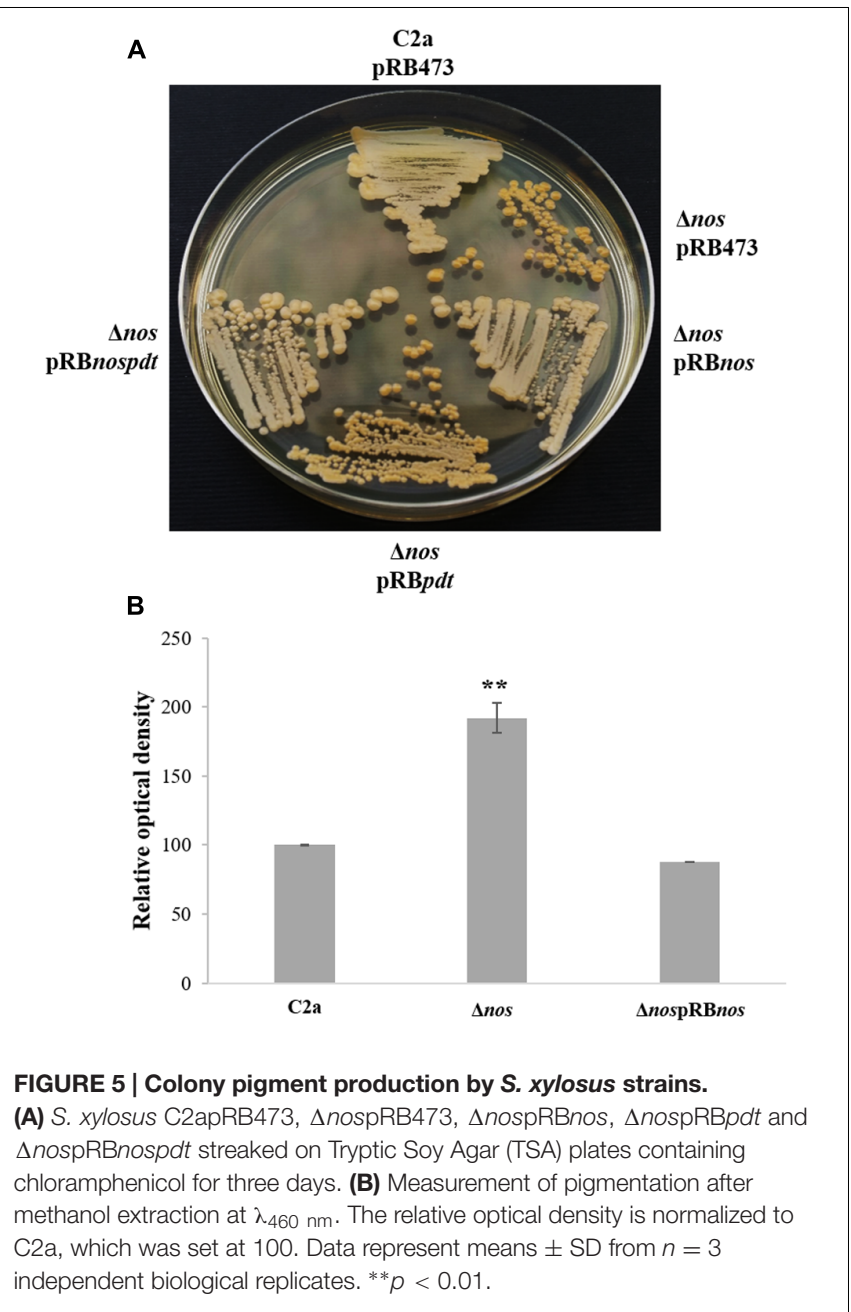

The katA gene was overexpressed 6- and 10-fold at $6 \mathrm{~h}$ and $24 \mathrm{~h}$, respectively in the $\Delta$ nos mutant related to the wild type (Figure 7). Expression of the two genes $k a t B$ and $k a t C$ was not modified in the $\Delta$ nos mutant at $6 \mathrm{~h}$ and was sharply downregulated at $24 \mathrm{~h}$ by comparison with the wild type (Figure 7). In the nos complemented mutant, only the nos gene was highly overexpressed at the two times of incubation, while the expression of the three catalase genes was not modified by comparison with the wild type. However, there was no difference in survival between the wild type and the nos complemented $\Delta$ nos mutant when treated with $200 \mathrm{mM} \mathrm{H}_{2} \mathrm{O}_{2}$ (4 log reduction) and $250 \mathrm{mM}$ (6 log reduction).

\section{DISCUSSION}

Staphylococcus xylosus is commonly used as a starter culture for fermented meat products, mainly because it reduces nitrate to nitrite, which undergoes chemical reactions leading to NO, which is responsible for color development (Talon and Leroy, 2006). As the safety of nitrite as a food additive is questioned, we studied the ability of $S$. xylosus to produce NO via a

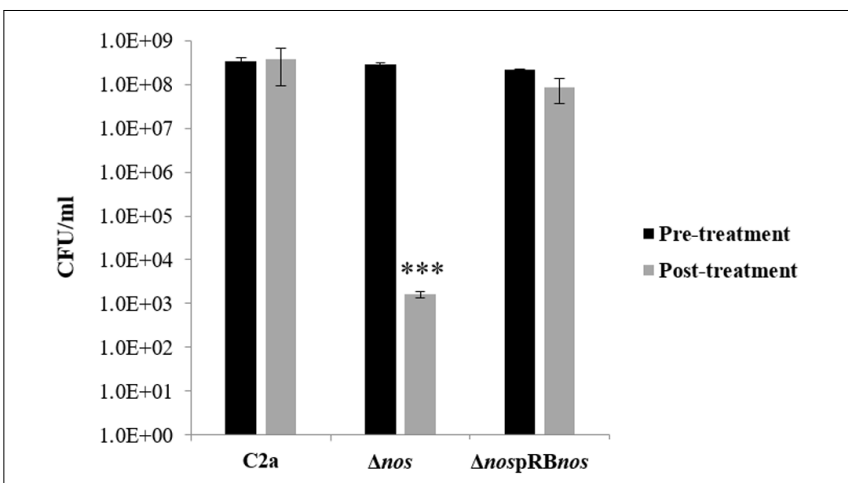

FIGURE 6 | Effect of nos deletion on sensitivity to oxidative stress. S. xylosus C2a, $\Delta$ nos, and $\Delta$ nospRBnos strains were grown under aerobic conditions up to $\mathrm{OD}=1$ and treated for $1 \mathrm{~h}$ with $150 \mathrm{mM}$ hydrogen peroxide $\left(\mathrm{H}_{2} \mathrm{O}_{2}\right)$. Cells, before and after treatment, were enumerated. Data represent means $\pm \mathrm{SD}$ from $n=2$ independent biological replicates. ${ }^{*} p<0.05$.

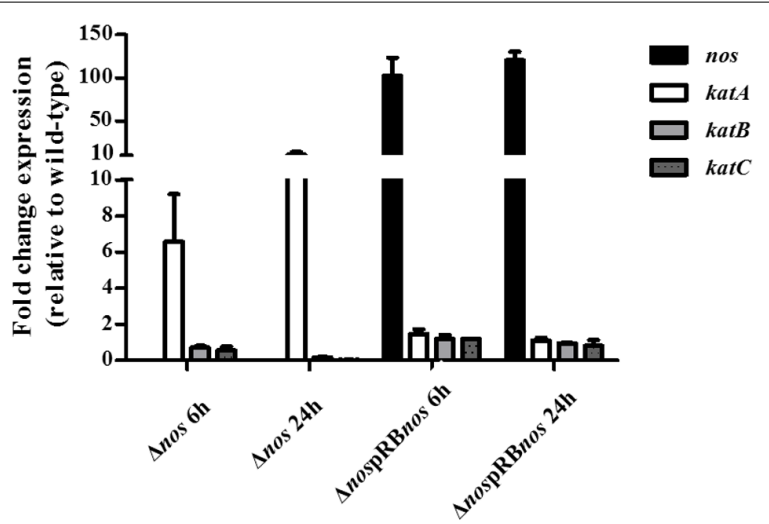

FIGURE 7 | Expression of nos and kat genes. S. xylosus C2a, $\Delta$ nos, and $\Delta$ nospRBnos strains were grown in TSB under aerobic conditions for 6 and $24 \mathrm{~h}$. Expression of nos and kat genes was evaluated by qRT-PCR. The Livak method $\left(2^{-\Delta \Delta C t}\right)$ was applied to analyze expression, using sod as reference gene. Results are expressed as means \pm SD from $n=3$ independent biological replicates.

pathway catalyzed by NOS. To demonstrate the production of $\mathrm{NO}$ by $S$. xylosus, we used the property of $\mathrm{NO}$ to form a $\mathrm{NO}$ complex of $\mathrm{Fe}(\mathrm{II})$ myoglobin, called nitrosomyoglobin. In previous studies $S$. xylosus FAX-1 and $S$. xylosus A1 were able to convert metmyoglobin to nitrosomyoglobin in vitro and in raw meat batter (Morita et al., 1998; Li et al., 2013, 2016). However, the mechanism of such NO production was not established. Here we demonstrate that NOS activity of S. xylosus led to production of NO. Under limited oxygenation, the $\Delta$ nos mutant remained able to form nitrosoheme (about 35-40\% related to the wild-type level). The $\Delta$ nos mutant was unable to produce NO under aerobic conditions. The loss of NOS activity in S. aureus UAMS-1 mutant led to NO production of $65 \%$ as evaluated using fluorescent stain DAF-FM diacetate after growth on agar plates (Sapp et al., 2014), while the deletion of nos in S. aureus UAMS1182 completely abolished NO production after growth under aerobic conditions (van Sorge et al., 2013). These results revealed that 
some Staphylococcus strains are able to produce NO by other pathways under limited oxygenation. NO production can be achieved via molybdoenzymes, which are recognized for their ability to catalyze reduction of nitrite to NO radical (Maia and Moura, 2015). The molybdenum-containing respiratory nitrate reductase is believed to be an important source of NO (Maia and Moura, 2015). S. xylosus C2a has a nitrate reductase that was previously shown to be expressed and active under static conditions (Talon et al., 1999). The involvement of NOS under aerobic conditions seems relevant to the oxygen requirement of NOS activity (Crane et al., 2010). The potential for NOS activity has been investigated in 86 strains belonging to $17 \mathrm{CNS}$ species (Sánchez-Mainar et al., 2014). Only one strain of S. haemolyticus showed NOS activity, based on its ability to produce L-citrulline in a meat-simulating medium supplemented with arginine under aerobic conditions (Sánchez-Mainar et al., 2014).

The deletion of nos in S. xylosus C2a slightly affected growth in complex medium under aerobic but not under microaerobic conditions. This nos deletion also increased $\mathrm{H}_{2} \mathrm{O}_{2}$ susceptibility. Such results are in accordance with previous studies showing that NO production through NOS activity protects Bacillus and $S$. aureus against oxidative stress generated by $\mathrm{H}_{2} \mathrm{O}_{2}$ exposure (Gusarov and Nudler, 2005; Shatalin et al., 2008; van Sorge et al., 2013). In B. subtilis, exogenous or endogenous NO protected cells from oxidative stress by boosting the activity of KatA, the major catalase (Gusarov and Nudler, 2005). Also, while sodA expression increased sharply in wild-type cells of $B$. subtilis during exponential growth, it was abolished in the $\Delta$ nos mutant (Gusarov et al., 2009). In S. xylosus C2a $\Delta$ nos mutant, the loss of NOS activity resulted in modulation of the expression of genes encoding catalases with upregulation of kat $A$ and downregulation of $k a t B$ and $k a t C$. We showed in a previous study that $S$. xylosus responded to nitrosative stress generated by nitrite in a meat model, notably by the upregulation of katB and $k a t C$ under the probable control of the repressor PerR and the downregulation of katA, which was not under PerR control (Vermassen et al., 2014). In this study, the limited amount of endogenous NO produced by the $\Delta$ nos mutant may not derepress PerR, contributing to the downregulation of katB and $k a t C$. In contrast to B. subtilis, expression of the gene $\operatorname{sodA}$ was not modified in the $S$. xylosus $\Delta$ nos mutant ( $\operatorname{sod}$ was used as housekeeping gene in our study).

The S. xylosus C2a $\Delta$ nos mutant displayed higher colony pigmentation than the wild-type strain after prolonged cultivation on agar medium, as observed for the $S$. aureus $\Delta$ nos strain UAMS-1 (Sapp et al., 2014). S. aureus produced the intermediary yellow carotenoid $4,4^{\prime}$-diaponeurosporene, which, after prolonged cultivation, is converted to the yellow-orange end-product staphyloxanthin (Wieland et al., 1994). The yellow pigment in $S$. xylosus is likely to be a carotenoid pigment with peak absorbance at $460 \mathrm{~nm}$. The carotenoid pigment of S. aureus protects against reactive oxygen species, so a non-pigmented mutant is more susceptible to oxidative killing (Clauditz et al., 2006). The increased colony pigmentation of the S. xylosus C2a $\Delta$ nos mutant could be a mechanism to cope with oxidative stress in conditions of extended incubation only on agar medium, as no pigmentation was observed in liquid medium.
The $S$. aureus nos gene is co-transcribed with the $p d t$ gene encoding a prephenate dehydratase, which catalyzes the penultimate reaction in phenylalanine biosynthesis (Sapp et al., 2014). This genetic arrangement is present in all publically available staphylococcal genome sequences and seems to be unique to the Staphylococcus genus (Sapp et al., 2014). As anticipated, co-transcription of the nos-pdt cluster was also demonstrated in S. xylosus. However, the role of the NOS and PDT enzymes of $S$. xylosus C2a did not appear to be interlinked. PDT was essential for $S$. xylosus growth only in the absence of phenylalanine, while NOS was not required to sustain phenylalanine biosynthesis.

\section{CONCLUSION}

Our results demonstrate NOS-dependent NO production in a coagulase-negative Staphylococcus. This endogenous NO production contributed to growth under aerobic conditions and to the cytoprotective effect against oxidative stress. S. xylosus is a species usually used as a starter culture in meat products. Therefore, NOS-dependent NO production in S. xylosus needs to be further characterized in meat products and optimized as a potential alternative to nitrate and nitrite in these products.

\section{AUTHOR CONTRIBUTIONS}

SL and RT conceived the study. GR, SL and RT designed the experiments and wrote the manuscript. GR performed the laboratory experiments. GR, VZ, PD, TS, SL and RT analyzed data. All the authors contributed to preparing the final version of the manuscript, read and approved the final manuscript.

\section{ACKNOWLEDGMENTS}

GR is a Ph.D. Research Fellow funded by ANRT, CIFRE 2013/1490. We would like to thank Philippe Gatellier, INRA, for helpful discussions about nitrosoheme pigment, and JeanPaul Chacornac and Carine Andant for technical assistance. The authors are grateful to David Marsh for correcting our English.

\section{SUPPLEMENTARY MATERIAL}

The Supplementary Material for this article can be found online at: http://journal.frontiersin.org/article/10.3389/fmicb.2017. 00598/full\#supplementary-material

FIGURE S1 | Growth of Staphylococcus xylosus strains in the Bioscreen Assay. S. xylosus C2a, $\Delta$ nos, and $\Delta$ nospRBnos in TSB. Data represent means $\pm \mathrm{SD}$ from $n=3$ independent biological replicates.

FIGURE S2 | Genetic cluster nos-pdt of S. xylosus C2a. The cluster of the two genes nos-pdt is depicted with primers used for PCR co-transcription.

FIGURE S3 | Impact of stresses on growth under microaerobic conditions of $\mathbf{S}$. xylosus strains. (A) Impact of salt and (B) impact of acid stresses. Data represent means \pm SD from $n=3$ independent biological replicates. 


\section{REFERENCES}

Alderton, W. K., Cooper, C. E., and Knowles, R. G. (2001). Nitric oxide synthases: structure, function and inhibition. Biochem. J. 357(Pt 3), 593-615. doi: 10.1042/ bj3570593

Barrière, C., Brückner, R., Centeno, D., and Talon, R. (2002). Characterisation of the katA gene encoding a catalase and evidence for at least a second catalase activity in Staphylococcus xylosus, bacteria used in food fermentation. FEMS Microbiol. Lett. 216, 277-283. doi: 10.1016/S0378-1097(02) 01030-3

Brückner, R. (1992). A series of shuttle vectors for Bacillus subtilis and Escherichia coli. Gene 122, 187-192. doi: 10.1016/0378-1119(92)90048-T

Brückner, R. (1997). Gene replacement in Staphylococcus carnosus and Staphylococcus xylosus. FEMS Microbiol. Lett. 151, 1-8. doi: 10.1016/S03781097(97)00116-X

Clauditz, A., Resch, A., Wieland, K. P., Peschel, A., and Götz, F. (2006). Staphyloxanthin plays a role in the fitness of Staphylococcus aureus and its ability to cope with oxidative stress. Infect. Immun. 74, 4950-4953. doi: 10.1128/ IAI.00204-06

Code of Federal Regulations (2016). Title 21 - Food and Drugs, Chapter I - Food and Drug Admistration, Department of Health and Human Services, Subchapter B - Food for Human Consumption, Part 172 - Food Additives Permitted for Direct Addition to Food for Human Consumption, Subpart B - Food Preservatives. Silver Spring, MD: U.S. Food and Drug Administration.

Commission Regulation [EU] (2011). No 1129/2011 of November 2011 amending Annex II to Regulation (EC) No 1333/2008 of the European Parliament and of the Council by establishing a Union list of food additives. Off. J. Eur. Union L295, 1-177.

Crane, B. R., Sudhamsu, J., and Patel, B. A. (2010). Bacterial nitric oxide synthases. Annu. Rev. Biochem. 79, 445-470. doi: 10.1146/annurev-biochem-062608103436

Fiegler, H., and Brückner, R. (1997). Identification of the serine acetyltransferase gene of Staphylococcus xylosus. FEMS Microbiol. Lett. 148, 181-187. doi: 10.1111/j.1574-6968.1997.tb10286.x

Gøtterup, J., Olsen, K., Knöchel, S., Tjener, K., Stahnke, L. H., and Møller, J. K. (2007). Relationship between nitrate/nitrite reductase activities in meat associated staphylococci and nitrosomyoglobin formation in a cured meat model system. Int. J. Food Microbiol. 120, 303-310. doi: 10.1016/j.ijfoodmicro. 2007.08.034

Gøtterup, J., Olsen, K., Knöchel, S., Tjener, K., Stahnke, L. H., and Møller, J. K. (2008). Colour formation in fermented sausages by meat-associated staphylococci with different nitrite- and nitrate-reductase activities. Meat Sci. 78, 492-501. doi: 10.1016/j.meatsci.2007.07.023

Götz, F., Zabielski, J., Philipson, L., and Lindberg, M. (1983). DNA homology between the arsenate resistance plasmid pSX267 from Staphylococcus xylosus and the penicillinase plasmid pI258 from Staphylococcus aureus. Plasmid 9, 126-137. doi: 10.1016/0147-619X(83)90015-X

Gündoğdu, A. K., Karahan, A. G., and Çakmakçi, M. L. (2006). Production of nitric oxide (NO) by lactic acid bacteria isolated from fermented products. Eur. Food Res. Technol. 223, 35-38. doi: 10.1007/s00217-0050097-8

Gusarov, I., and Nudler, E. (2005). NO-mediated cytoprotection: instant adaptation to oxidative stress in bacteria. Proc. Natl. Acad. Sci. U.S.A. 102, 13855-13860. doi: $10.1073 /$ pnas.0504307102

Gusarov, I., Shatalin, K., Starodubtseva, M., and Nudler, E. (2009). Endogenous nitric oxide protects bacteria against a wide spectrum of antibiotics. Science 325, 1380-1384. doi: 10.1126/science.1175439

Gusarov, I., Starodubtseva, M., Wang, Z. Q., McQuade, L., Lippard, S. J., Stuehr, D. J., et al. (2008). Bacterial nitric-oxide synthases operate without a dedicated redox partner. J. Biol. Chem. 283, 13140-13147. doi: 10.1074/jbc.M71017 8200

Honikel, K. O. (2008). The use and control of nitrate and nitrite for the processing of meat products. Meat Sci. 78, 68-76. doi: 10.1016/j.meatsci.2007. 05.030

Kabir, M., Sudhamsu, J., Crane, B. R., Yeh, S. R., and Rousseau, D. L. (2008). Substrate-ligand interactions in Geobacillus stearothermophilus nitric oxide synthase. Biochemistry 47, 12389-12397. doi: 10.1021/bi801491e
Kers, J. A., Wach, M. J., Krasnoff, S. B., Widom, J., Cameron, K. D., Bukhalid, R. A., et al. (2004). Nitration of a peptide phytotoxin by bacterial nitric oxide synthase. Nature 429, 79-82. doi: 10.1038/nature02504nature02504

Li, P., Kong, B., Chen, Q., Zheng, D., and Liu, N. (2013). Formation and identification of nitrosomyoglobin by Staphylococcus xylosus in raw meat batters: a potential solution for nitrite substitution in meat products. Meat Sci. 93, 67-72. doi: 10.1016/j.meatsci.2012.08.003

Li, P. J., Luo, H. T., Kong, B. H., Liu, Q., and Chen, C. G. (2016). Formation of red myoglobin derivatives and inhibition of spoilage bacteria in raw meat batters by lactic acid bacteria and Staphylococcus xylosus. LWT Food Sci. Technol. 68, 251-257. doi: 10.1016/j.lwt.2015.12.035

Livak, K. J., and Schmittgen, T. D. (2001). Analysis of relative gene expression data using real-time quantitative PCR and the 2(-Delta Delta C(T)) Method. Methods 25, 402-408. doi: 10.1006/meth.2001.1262

Maia, L. B., and Moura, J. J. (2015). Nitrite reduction by molybdoenzymes: a new class of nitric oxide-forming nitrite reductases. J. Biol. Inorg. Chem. 20, 403-433. doi: 10.1007/s00775-014-1234-2

Montgomery, H. J., Dupont, A. L., Leivo, H. E., and Guillemette, J. G. (2010). Cloning, expression, and purification of a nitric oxide synthase-like protein from Bacillus cereus. Biochem. Res. Int. 2010:489892. doi: 10.1155/2010/ 489892

Morikawa, K., Maruyama, A., Inose, Y., Higashide, M., Hayashi, H., and Ohta, T. (2001). Overexpression of sigma factor, sigma(B), urges Staphylococcus aureus to thicken the cell wall and to resist beta-lactams. Biochem. Biophys. Res. Commun. 288, 385-389. doi: 10.1006/bbrc.2001.5774

Morita, H., Sakata, R., and Nagata, Y. (1998). Nitric oxide complex of iron(II) myoglobin converted from metmyoglobin by Staphylococcus xylosus. J. Food Sci. 63, 352-355. doi: 10.1111/j.1365-2621.1998.tb15740.x

Patel, B. A., Moreau, M., Widom, J., Chen, H., Yin, L., Hua, Y., et al. (2009). Endogenous nitric oxide regulates the recovery of the radiation-resistant bacterium Deinococcus radiodurans from exposure to UV light. Proc. Natl. Acad. Sci. U.S.A 106, 18183-18188. doi: 10.1073/pnas.0907262106

Sánchez-Mainar, M., Weckx, S., and Leroy, F. (2014). Coagulase-negative Staphylococci favor conversion of arginine into ornithine despite a widespread genetic potential for nitric oxide synthase activity. Appl. Environ. Microbiol. 80, 7741-7751. doi: 10.1128/AEM.02298-14

Sapp, A. M., Mogen, A. B., Almand, E. A., Rivera, F. E., Shaw, L. N., Richardson, A. R., et al. (2014). Contribution of the nos-pdt operon to virulence phenotypes in methicillin-sensitive Staphylococcus aureus. PLoS ONE 9:e108868. doi: 10.1371/journal.pone.0108868

Shatalin, K., Gusarov, I., Avetissova, E., Shatalina, Y., McQuade, L. E., Lippard, S. J., et al. (2008). Bacillus anthracis-derived nitric oxide is essential for pathogen virulence and survival in macrophages. Proc. Natl. Acad. Sci. U.S.A. 105, 1009-1013. doi: 10.1073/pnas.0710950105

Talon, R., and Leroy, S. (2006). "Chapter 16-Latest developments in meat bacterial starters," in Advanced Technologies for Meat Processing, eds L. M. L. Nollet and F. Toldra (New York, NY: CRC Press, Taylor and Francis group), 401-418.

Talon, R., Walter, D., Chartier, S., Barrière, C., and Montel, M. C. (1999). Effect of nitrate and incubation conditions on the production of catalase and nitrate reductase by staphylococci. Int. J. Food Microbiol. 52, 47-56. doi: 10.1016/ S0168-1605(99)00127-0

Vaish, M., and Singh, V. K. (2013). Antioxidant functions of nitric oxide synthase in a methicillin sensitive Staphylococcus aureus. Int. J. Microbiol. 2013:312146. doi: $10.1155 / 2013 / 312146$

van Sorge, N. M., Beasley, F. C., Gusarov, I., Gonzalez, D. J., von KöckritzBlickwede, M., Anik, S., et al. (2013). Methicillin-resistant Staphylococcus aureus bacterial nitric-oxide synthase affects antibiotic sensitivity and skin abscess development. J. Biol. Chem. 288, 6417-6426. doi: 10.1074/jbc.M112. 448738

Vermassen, A., de la Foye, A., Loux, V., Talon, R., and Leroy, S. (2014). Transcriptomic analysis of Staphylococcus xylosus in the presence of nitrate and nitrite in meat reveals its response to nitrosative stress. Front. Microbiol. 5:691. doi: $10.3389 /$ fmicb.2014.00691

Wieland, B., Feil, C., Gloria-Maercker, E., Thumm, G., Lechner, M., Bravo, J. M., et al. (1994). Genetic and biochemical analyses of the biosynthesis of the yellow carotenoid 4,4'-diaponeurosporene of Staphylococcus aureus. J. Bacteriol. 176, 7719-7726. doi: 10.1128/jb.176.24.7719-7726.1994 
Yu, C., Jiao, J., Ma, L., and Sun, W. (2016). Effect of pH on the stability and molecular structure of nitrosyl hemochromogen. Food Chem. 196, 503-508. doi: 10.1016/j.foodchem.2015.09.067

Conflict of Interest Statement: The authors declare that the research was conducted in the absence of any commercial or financial relationships that could be construed as a potential conflict of interest.
Copyright $\odot 2017$ Ras, Zuliani, Derkx, Seibert, Leroy and Talon. This is an openaccess article distributed under the terms of the Creative Commons Attribution License (CC BY). The use, distribution or reproduction in other forums is permitted, provided the original author(s) or licensor are credited and that the original publication in this journal is cited, in accordance with accepted academic practice. No use, distribution or reproduction is permitted which does not comply with these terms. 